Quim. Nova, Vol. 34, No. 3, 434-438, 2011

\title{
SÍNTESE HIDROTERMAL ASSISTIDA POR MICRO-ONDAS COMO METODOLOGIA SINTÉTICA EFICIENTE PARA OBTENÇÃO DA REDE METALORGÂNICA [Zn(BDC)( $\left.\left.\mathrm{H}_{2} \mathrm{O}\right)_{2}\right]_{n}$
}

\author{
Kaline A. Wanderley* e Severino Alves Júnior \\ Departamento de Química Fundamental, Universidade Federal de Pernambuco, Av. Luis Freire, s/n, 50590-470 Recife - PE, Brasil \\ Carlos de Oliveira Paiva-Santos \\ Departamento de Físico-Química, Instituto de Química, Universidade Estadual Paulista, CP 355, 14801-970 Araraquara - SP, Brasil
}

Recebido em 28/5/10; aceito em 24/10/10; publicado na web em 26/1/11

\begin{abstract}
MICROWAVE-ASSISTED HYDROTHERMAL SYNTHESIS AS AN EFFICIENT METHOD FOR OBTAINING $\left[\mathrm{Zn}(\mathrm{BDC})\left(\mathrm{H}_{2} \mathrm{O}\right)_{2}\right]_{n}$ METAL-ORGANIC FRAMEWORK. This work presents the optimization of the microwave-assisted hydrothermal synthesis of $\left[\mathrm{Zn}(\mathrm{BDC})\left(\mathrm{H}_{2} \mathrm{O}\right)_{2}\right]_{n}$. The reactions were carried out at the fixed temperature of $120{ }^{\circ} \mathrm{C}$ for $10,20,30$ and 40 min. Pure crystalline $\left[\mathrm{Zn}(\mathrm{BDC})\left(\mathrm{H}_{2} \mathrm{O}\right)_{2}\right]_{n}$ was obtained in high yield $(\mathrm{ca} .90 \%)$ with a reaction time of $10 \mathrm{~min}$. The phase obtained and its purity was confirmed by Rietveld refinement, with a final value for $\mathrm{R}_{\mathrm{wp}} / \mathrm{R}_{\mathrm{exp}}$ equal to 1.48 . Increased reaction times (20,30 and 40 min) favored the formation of unwanted by products, resulting in mixtures of several crystalline phases.
\end{abstract}

Keywords: metal-organic framework (MOF); microwave; Rietveld refinement.

\section{INTRODUÇÃO}

As redes metalorgânicas são uma extensa classe de materiais cristalinos que apresentam geralmente um átomo central (íon metálico) coordenado a moléculas orgânicas (ligantes), em que essa unidade metal-ligante se repete formando uma estrutura polimérica de complexos metálicos. Devido à sua grande estabilidade, estruturas cristalinas bem definidas, elevada especificidade na obtenção de diferentes compostos e ampla funcionalidade orgânica devido à escolha do ligante orgânico e íon metálico, esses materiais têm se destacado há alguns anos como uma importante interface entre a ciência de materiais e a química sintética. ${ }^{1-4}$

As propriedades das redes metalorgânicas dependem parcialmente das propriedades dos ligantes orgânicos e da forma com que ele está coordenado ao metal. Assim, novas propriedades podem ser dadas a estes materiais através de alterações prévias do ligante orgânico obtido por diferentes rotas sintéticas. O desenvolvimento de rotas econômicas e seguras para sínteses de materiais metalorgânicos tem sido uma tarefa desafiadora para aplicações práticas. Esses materiais são predominantemente sintetizados sob condições solvotermais ou via cristalização à temperatura ambiente, levando dias ou até semanas para cristalizar. ${ }^{5,6}$ Geralmente, sínteses em fornos de micro-ondas têm uma drástica redução no tempo reacional e sua simplicidade e eficiência energética no processo de aquecimento têm se tornado um fator preponderante na escolha do método sintético. ${ }^{7,8}$

$\mathrm{O}$ método de irradiação de micro-ondas tem sido estudado em sínteses não apenas de moléculas orgânicas, ${ }^{9}$ mas também de materiais inorgânicos, incluindo várias zeólitas, ${ }^{10,11}$ peneiras moleculares e nanopartículas. ${ }^{12,13}$ Devido ao curto tempo gasto durante o processo de cristalização desses materiais, sínteses por micro-ondas provêm métodos sintéticos de materiais porosos com economia de tempo de reação dentro de alguns minutos, oferecendo seletividade de etapas e facilidade no controle da morfologia. ${ }^{14}$ Estudos nesta área promovem um amplo campo interdisciplinar que vem crescendo rápido e

\footnotetext{
*e-mail: ka_wanderley@yahoo.com.br
}

constantemente, uma vez que as redes metalorgânicas apresentam promissoras aplicações em armazenamento, separação e sorção de gases, ${ }^{15,16}$ catálise, ${ }^{17,18}$ liberação de fármacos, ${ }^{19,20}$ fases estacionárias para cromatografia, ${ }^{21,22}$ entre outras.

Uma série de ligantes têm sido propostos e utilizados na síntese de novos materiais metalorgânicos, dentre os quais, os policarboxilatos aromáticos são particularmente interessantes devido à robustez química e propriedades fotofísicas. ${ }^{23}$ Estruturas metalorgânicas contendo zinco e ácido 1,4-benzenodicarboxílico (1,4- $\left.\mathrm{H}_{2} \mathrm{BDC}\right)$ como ligante têm sido extensivamente estudados, sendo os trabalhos de Yaghi e colaboradores os mais relevantes. ${ }^{24-27} \mathrm{O}$ ácido 1,4- $\mathrm{H}_{2} \mathrm{BDC}$ desempenha papel interessante na construção de redes metalorgânicas, uma vez que pode funcionar como ponte entre os centros metálicos e adotar diversos modos de coordenação, formando estruturas em uma, ${ }^{28}$ duas $^{29}$ ou três dimensões (1D, 2D e 3D). ${ }^{30}$

A rede metalorgânica $\left[\mathrm{Zn}(\mathrm{BDC})\left(\mathrm{H}_{2} \mathrm{O}\right)_{2}\right]_{n}$, contendo o 1,4-benzenodicarboxilato (BDC) em sua estrutura, foi obtida pela primeira vez por Guilera e colaboradores, em 1999, através do método de precipitação por 24 h. ${ }^{28}$ Em 2001, Edgar e colaboradores obtiveram o mesmo material através de transformações sólido-estruturais por tratamento térmico e adição de solventes. ${ }^{31}$ Em 2002, Li-Na Zhu e colaboradores obtiveram o $\left[\mathrm{Zn}(\mathrm{BDC})\left(\mathrm{H}_{2} \mathrm{O}\right)_{2}\right]_{n}$ através da reação entre sal de BDC e zinco em metanol por 30 min, obtendo cristais com $80 \%$ de rendimento. ${ }^{32}$ Entretanto, em nenhum dos referidos trabalhos os autores relatam a obtenção de uma fase cristalina pura.

Com a finalidade de se obter uma rede metalorgânica por uma metodologia mais simples e reprodutível, neste trabalho foi otimizada a síntese do $\left[\mathrm{Zn}(\mathrm{BDC})\left(\mathrm{H}_{2} \mathrm{O}\right)_{2}\right]_{n}$ já conhecida na literatura por uma rota sintética diferente do que foi reportado, através de síntese hidrotermal assistida por micro-ondas, uma metodologia mais rápida, fácil, eficiente e com maior reprodutibilidade. Através do método de irradiação de micro-ondas é possível sintetizar materiais em curto espaço de tempo, controlar a distribuição de tamanho das partículas, além da facilidade no controle dos parâmetros experimentais (temperatura, potência, pressão, tempo reacional). 


\section{PARTE EXPERIMENTAL}

\section{Materiais e reagentes}

Ácido 1,4-benzenodicarboxílico (1,4- $\left.\mathrm{H}_{2} \mathrm{BDC}\right)$ fornecido pela Terphane Ltda., $\mathrm{NaOH}$ anidro (97\%) adquirido da Dinâmica Química Ltda. e $\mathrm{Zn}\left(\mathrm{NO}_{3}\right)_{2} \cdot 6 \mathrm{H}_{2} \mathrm{O}$ (98\%) fornecido pela Sigma-Aldrich foram usados sem purificação prévia para a síntese da rede metalorgânica.

\section{Síntese do $\mathrm{Na}_{2} \mathrm{BDC}$ e da rede metalorgânica $\left[\mathrm{Zn}(\mathrm{BDC})\left(\mathrm{H}_{2} \mathrm{O}\right)_{2}\right]_{\mathrm{n}}$} $\mathrm{Na}_{2} \mathrm{BDC}$

Uma quantidade conhecida de $\mathrm{NaOH}(20 \mathrm{mmol} ; 0,8 \mathrm{~g})$ foi dissolvida em $\mathrm{H}_{2} \mathrm{O}(10 \mathrm{~mL})$ e adicionada lentamente ao 1,4- $\mathrm{H}_{2} \mathrm{BDC}$ (10 mmol; 1,66 g) em $\mathrm{H}_{2} \mathrm{O}$ (aproximadamente $50 \mathrm{~mL}$ ). Após a dissolução da mistura de $\mathrm{NaOH}$ e $1,4-\mathrm{H}_{2} \mathrm{BDC}$ em $\mathrm{H}_{2} \mathrm{O}$ sob agitação, evaporou-se cerca de um terço do volume total de água $(20 \mathrm{~mL})$ e adicionou-se $20 \mathrm{~mL}$ de etanol até precipitação do $\mathrm{Na}_{2} \mathrm{BDC}$. O precipitado (Equação 1) foi filtrado e secado à temperatura ambiente, obtendo-se um rendimento de aproximadamente $88 \%$ através de cálculo estequiométrico.

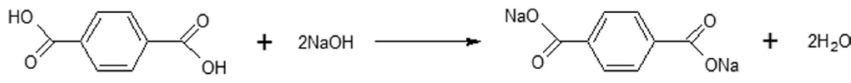

$\left[\mathrm{Zn}(\mathrm{BDC})\left(\mathrm{H}_{2} \mathrm{O}\right)_{2}\right]_{n}$

Reatores de $10 \mathrm{~mL}$ IntelliVent para reações em micro-ondas foram preenchidos com misturas de $\mathrm{Na}_{2} \mathrm{BDC}(0,25 \mathrm{mmol} ; 0,053 \mathrm{~g})$, $\mathrm{Zn}\left(\mathrm{NO}_{3}\right)_{2} \cdot 6 \mathrm{H}_{2} \mathrm{O}(0,5 \mathrm{mmol} ; 0,148 \mathrm{~g})$ e $\mathrm{H}_{2} \mathrm{O}(4 \mathrm{~mL})$; cada mistura foi agitada por 15 min para garantir a homogeneização do sistema e os reatores colocados individualmente dentro de um micro-ondas CEM Focused Microwave ${ }^{\mathrm{TM}}$ Synthesis System Discover S-Class por diferentes tempos para cada reator $(10,20,30$ e $40 \mathrm{~min})$ e a uma mesma temperatura de $120{ }^{\circ} \mathrm{C}$ sob constante agitação.

A potência máxima do micro-ondas foi $60 \mathrm{~W}$ para garantir que a temperatura de $120{ }^{\circ} \mathrm{C}$ seria atingida nos tempos de reação desejados (10, 20, 30 e 40 min) e a pressão variando em função da potência, de acordo com o equipamento. A temperatura e a pressão foram controladas dentro do reator e o fluxo constante de ar (cerca de 10 bar de pressão) garantiu um controle rigoroso das condições das sínteses.

Após a reação hidrotermal assistida por micro-ondas, os materiais foram resfriados à temperatura ambiente, filtrados sob vácuo e lavados com água e acetona, respectivamente. Os produtos foram coletados e secos ao ar. A fase pura (Equação 2) de coloração branca foi obtida apenas para a síntese assistida por $10 \min (0,061 \mathrm{~g}, 90 \%$ de rendimento em relação ao ligante).

$n N_{2} \mathrm{BDC}+\mathrm{nZn}\left(\mathrm{NO}_{3}\right)_{2} \cdot 6 \mathrm{H}_{2} \mathrm{O} \longrightarrow\left[\mathrm{Zn}(\mathrm{BDC})\left(\mathrm{H}_{2} \mathrm{O}\right)_{2}\right]_{n}+2 \mathrm{nHNO}_{3}+2 \mathrm{nNaOH}+2 \mathrm{nH}_{2} \mathrm{O}$

\section{Análise elementar}

Análises elementares de carbono, nitrogênio, hidrogênio e enxofre (CNHS) foram realizadas em um analisador elementar $\mathrm{CE}$ Instruments, modelo EA1110.

\section{Microscopia eletrônica de varredura}

As imagens de microscopia eletrônica de varredura foram coletadas por deposição em alumínio revestido com carbono, usando um microscópio eletrônico de varredura SU-70 trabalhando a $15 \mathrm{kV}$.

\section{Difração de raios $X$ por policristais}

As medidas de difração de raios $\mathrm{X}$ dos materiais sintetizados com 10, 20, 30 e 40 min de irradiação de micro-ondas foram realizadas à temperatura ambiente em um difratômetro Philips X'Pert MPD, ânodo de cobre $\left(\lambda_{\mathrm{k \alpha 1}}=1,540598 \AA\right.$ e $\left.\lambda_{\mathrm{k} \alpha 22}=1,544426 \AA\right)$, potência no tubo de $2 \mathrm{~kW}$ ( $40 \mathrm{kV}$ de radiação $\mathrm{X}, 50 \mathrm{~mA}$ ), equipado com um detector $\mathrm{X}^{\prime}$ Celerator e configuração óptica Bragg-Bretano. A intensidade foi coletada em modo de varredura contínua com $2 \theta$ entre 5 e $50^{\circ}$ e intensidades registradas a cada $\Delta 2 \theta=0,04^{\circ}$.

A rede metalorgânica $\left[\mathrm{Zn}(\mathrm{BDC})\left(\mathrm{H}_{2} \mathrm{O}\right)_{2}\right]_{n}$ foi caracterizada por difração de raios $\mathrm{X}$ de pós, as medidas foram realizadas à temperatura ambiente em um difratômetro Rigaku RINT2000 com ânodo rotatório de cobre $\left(\lambda_{\mathrm{k} \alpha 1}=1,5404 \AA, \lambda_{\mathrm{k} \alpha 2}=1,5444 \AA \mathrm{I}_{\alpha 2} / \mathrm{I}_{\alpha 1}=0,5\right)$. A intensidade foi coletada através do método passo a passo num intervalo de $2 \theta$ entre 10 e $50^{\circ}$, com $\Delta 2 \theta$ de $0,02^{\circ}$, fenda Soller de $2,5^{\circ}$ de divergência, fenda de espalhamento de $0,5^{\circ}$ e fenda de recepção de $0,3 \mathrm{~mm}$. Para o refinamento pelo método de Rietveld ${ }^{33}$ foi usado o programa $\mathrm{GSAS}^{34}$ e o modelo da estrutura cristalina descrito por Guilera e colaboradores.$^{28} \mathrm{~A}$ orientação preferencial foi corrigida pelo modelo dos esféricos harmônicos descrito por Jarvinen. ${ }^{35}$ Para o ajuste do perfil dos picos de difração foi usada a função de Thompson-CoxHastings ${ }^{36}$ modificada por Young e Desai. ${ }^{37}$

\section{Espectroscopia de absorção na região do infravermelho}

As análises qualitativa e quantitativa dos modos vibracionais na região do infravermelho de 4000 a $400 \mathrm{~cm}^{-1}$ do $1,4-\mathrm{H}_{2} \mathrm{BDC}, \mathrm{Na}_{2} \mathrm{BDC}$ e $\left[\mathrm{Zn}(\mathrm{BDC})\left(\mathrm{H}_{2} \mathrm{O}\right)_{2}\right]_{n}$ foram realizadas por infravermelho com transformada de Fourier usando um espectrômetro Bruker, modelo I'FS66.

\section{Análise termogravimétrica}

Os dados termogravimétricos foram obtidos a partir de 3,0 mg de cada amostra utilizando uma termobalança Shimadzu modelo TGA 50, em uma faixa de temperatura de 25 a $800{ }^{\circ} \mathrm{C}$, usando cadinho de platina sob atmosfera de nitrogênio $(50 \mathrm{~mL} / \mathrm{min})$ e taxa de aquecimento de $10^{\circ} \mathrm{C} / \mathrm{min}$.

\section{RESULTADOS E DISCUSSÃO}

As análises elementares do $\mathrm{Na}_{2} \mathrm{BDC}$ estavam bem de acordo com os valores calculados, podendo ser utilizado na síntese do $\left[\mathrm{Zn}(\mathrm{BDC})\left(\mathrm{H}_{2} \mathrm{O}\right)_{2}\right]_{n}$. Análise elementar calculada para $\mathrm{C}_{8} \mathrm{H}_{4} \mathrm{O}_{4} \mathrm{Na}_{2}$ (\%): C 45,69; H 1,92, encontrada (\%): C 45,32; H 1,80. Os padrões de difração de raios X para os materiais sintetizados com 10, 20, 30 e 40 min de irradiação de micro-ondas (Figura 1a) foram medidos a fim de se confirmar a obtenção unicamente da fase pura da rede metalorgânica $\left[\mathrm{Zn}(\mathrm{BDC})\left(\mathrm{H}_{2} \mathrm{O}\right)_{2}\right]_{n}$. Os resultados mostram que. com o aumento gradual do tempo reacional, se observa a formação de uma mistura de diferentes fases cristalinas, gerando um grau de impureza não desejado.

A obtenção do $\left[\mathrm{Zn}(\mathrm{BDC})\left(\mathrm{H}_{2} \mathrm{O}\right)_{2}\right]_{n}$ pôde ser confirmada através de refinamento de Rietveld apenas para a síntese realizada durante 10 min (Figura 1b). Os fatores de confiabilidade ${ }^{34,38} R_{w p}$ e $\chi^{2}$ no final do refinamento foram de $13,20 \%$ e 2,202, respectivamente. O valor esperado $^{34}$ de $\mathrm{R}_{\text {exp }}$ para esse refinamento $\left(R_{\text {exp }}=R_{w p} . \sqrt{ } x^{2}\right)$ é $8,9 \%$. A razão $R_{\text {wp }} / R_{\text {exp }}=1,48$ mostra que os valores obtidos dos índices indicam que o refinamento foi realizado adequadamente. Nota-se também na Figura 1b, que todos os picos foram ajustados por essa estrutura cristalina, indicando que apenas essa fase está presente na amostra. Os parâmetros de rede e dados de refinamento de Rietveld para o $\left[\mathrm{Zn}(\mathrm{BDC})\left(\mathrm{H}_{2} \mathrm{O}\right)_{2}\right]_{n}$ estão dispostos na Tabela 1 . 

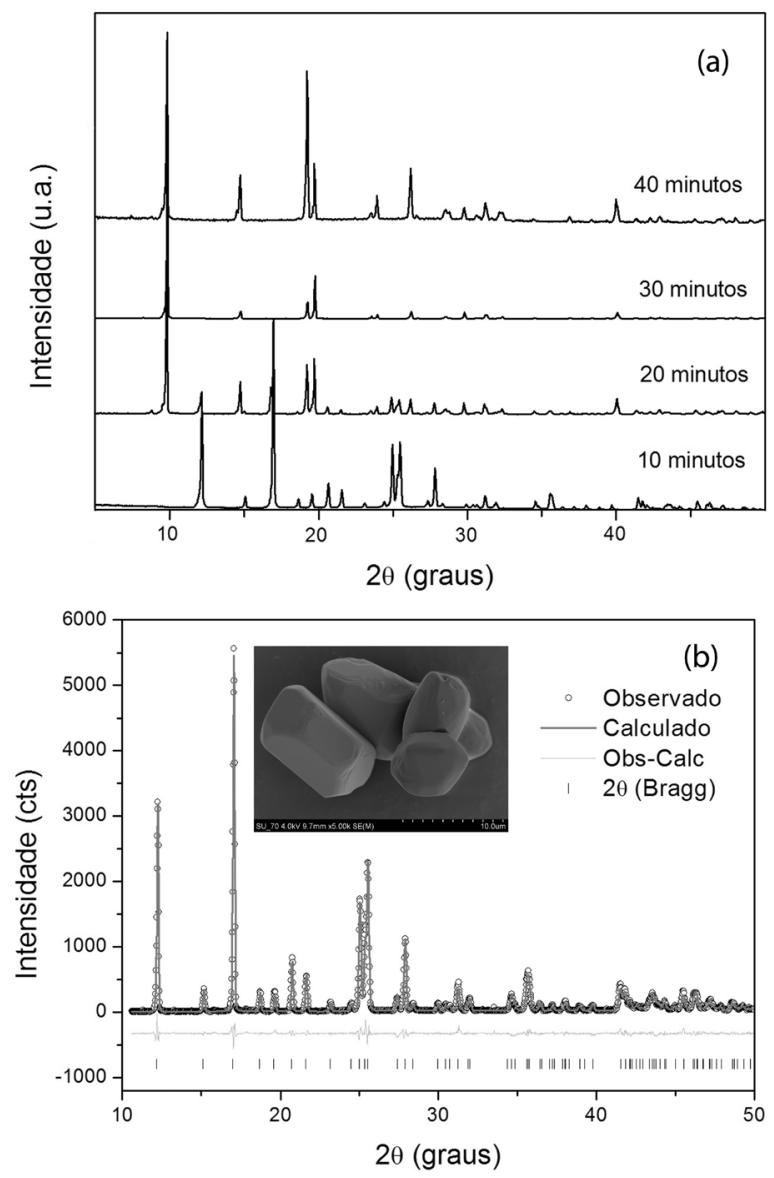

Figura 1. (a) Padrões de raios X para sínteses em 10, 2, 30 e 40 min por micro-ondas; (b) refinamento de Rietveld para a síntese do $\left[\mathrm{Zn}(\mathrm{BDC})\left(\mathrm{H}_{2} \mathrm{O}\right)_{2}\right]_{n}$ em 10 min e imagem de microscopia eletrônica de varredura ilustrando a morfologia do material

Tabela 1. Parâmetros de rede e dados do refinamento de Rietveld para o $\left[\mathrm{Zn}(\mathrm{BDC})\left(\mathrm{H}_{2} \mathrm{O}\right)_{2}\right]_{n}$ encontrados pelo GSAS

\begin{tabular}{cc}
\hline Fórmula empírica & $\mathrm{C}_{8} \mathrm{H}_{8} \mathrm{O}_{6} \mathrm{Zn}$ \\
\hline Peso molecular & 265,51 \\
Sistema cristalino & Monoclínico \\
Grupo espacial & $C 2 / c$ \\
$a$ & $14,9783(4) \AA$ \\
$b$ & $5,0247(2) \AA$ \\
$c$ & $12,0811(3) \AA$ \\
$\beta$ & $103,823(2)^{\circ}$ \\
Volume & $882,92(4) \AA$ \\
$\mathrm{Z}$ & 4 \\
Fatores de confiabilidade do refinamento de Rietveld \\
$\mathrm{R}_{\mathrm{p}}$ & $9,11 \%$ \\
$\mathrm{R}_{\mathrm{wp}}$ & $13,20 \%$ \\
$\mathrm{R}\left(\mathrm{F}^{2}\right)$ & $6,73 \%$ \\
$\chi^{2}$ & 2,202 \\
\hline
\end{tabular}

A metodologia sintética da rede metalorgânica $\left[\mathrm{Zn}(\mathrm{BDC})\left(\mathrm{H}_{2} \mathrm{O}\right)_{2}\right]_{n}$, reportada na literatura, ${ }^{28,31,32}$ foi modificada com o propósito de aumentar a cristalinidade total, bem como tornar a síntese do material mais rápida, prática e eficiente. A reação hidrotermal assistida por micro-ondas na razão molar 1:2 ligante-metal forneceu excelentes materiais com elevado rendimento. Análises elementares de CNHS estão bem de acordo com os respectivos valores calculados. Análise elementar calculada para $\mathrm{C}_{8} \mathrm{H}_{8} \mathrm{O}_{6} \mathrm{Zn}(\%)$ : C 36, 17; H 3,04, encontrada (\%): C 36,01; H 3,12. Monocristais com formas largas e bem definidas (tamanho do cristal na faixa de 50 a $100 \mu \mathrm{m}$ ) puderam ser isolados e revelados através de imagens de MEV (Figura 1b).

$\mathrm{O}\left[\mathrm{Zn}(\mathrm{BDC})\left(\mathrm{H}_{2} \mathrm{O}\right)_{2}\right]_{n}$ constituído por centros $\mathrm{Zn}^{2+}$ ligados a dois resíduos de $\mathrm{BDC}$ pelos átomos de oxigênios $\mathrm{O}(1)$ e $\mathrm{O}(1)^{\#}$ forma uma estrutura linear em 1D. A esfera de coordenação do cátion metálico é completada por duas moléculas de água, formando um poliedro descrito por um tetraedro distorcido. A Figura 2 mostra uma projeção do $\left[\mathrm{Zn}(\mathrm{BDC})\left(\mathrm{H}_{2} \mathrm{O}\right)_{2}\right]_{n}$ e o poliedro de coordenação do metal. As distâncias de ligação são Zn-O(1) 2,005(15) Å e Zn-O(2) 2,550(16) Å. Este fato sugere uma interação significativa entre o $\mathrm{Zn}^{2+}$ e o átomo de oxigênio $\mathrm{O}(2)$, porém não caracteriza a formação de uma ligação coordenada, uma vez que as distâncias de ligação M-O em ligantes carboxilatos atuando como quelatos são aproximadamente as mesmas. ${ }^{39,40}$ Desta forma, cada ligante BDC adota o modo de coordenação bis-monodentado (syn-anti) conectando dois $\mathrm{Zn}^{2+}$ adjacentes, formando uma cadeia 1D em zig-zag ao logo do plano cristalográfico $a c .^{28}$

(a)

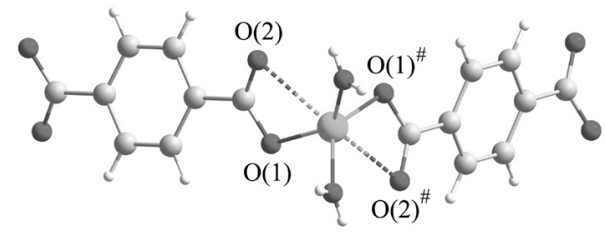

(b)

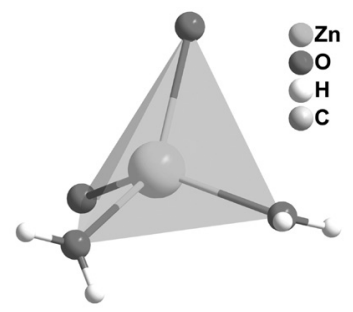

Figura 2. (a) Unidade assimétrica do $\left[\mathrm{Zn}(\mathrm{BDC})\left(\mathrm{H}_{2} \mathrm{O}\right)_{2}\right]_{n}$; (b) poliedro de coordenação do cátion $\mathrm{Zn}^{2+}$. A operação de simetria usada para gerar os átomos equivalentes: ${ }^{\#} 0,5+x, 1,5-y, 0,5+z$

A estrutura supramolecular do cristal (Figura 3) é formada pela conexão das cadeias poliméricas $1 \mathrm{D}$ por empacotamentos $\pi-\pi$ e por uma série de ligações de hidrogênio presente na estrutura. Os anéis aromáticos do ligante BDC das cadeias 1D emparelham-se perpendicularmente ao longo do plano cristalográfico $b$ com distância interlamelar de 5,0 Å. As séries de ligações de hidrogênio envolvem os átomos de oxigênio das moléculas de água coordenadas ao metal e os átomos de oxigênio $\mathrm{O}(2)$ simetricamente relacionados, oriundos dos resíduos de BDC. Para melhor visualização da estrutura supramolecular do $\left[\mathrm{Zn}(\mathrm{BDC})\left(\mathrm{H}_{2} \mathrm{O}\right)_{2}\right]_{n}$, consultar o material suplementar (Figura 1S).

Os espectros de IV obtidos estão dispostos na Figura 4. O espectro de infravermelho do 1,4- $\mathrm{H}_{2} \mathrm{BDC}$ apresenta uma deformação axial de $v(\mathrm{O}-\mathrm{H})$ larga de $3100 \mathrm{a} 2550 \mathrm{~cm}^{-1}$, aproximadamente. Apresenta também o sinal característico da carbonila, deformação axial de $v(C=O)$ carboxílica do dímero, próximo a $1685 \mathrm{~cm}^{-1}$. Apresenta deformação angular no plano de $v(\mathrm{C}-\mathrm{O}-\mathrm{H})$ em $1428 \mathrm{~cm}^{-1}$ e a deformação axial de $v(C-O)$, dímero, em $1283 \mathrm{~cm}^{-1}$. 


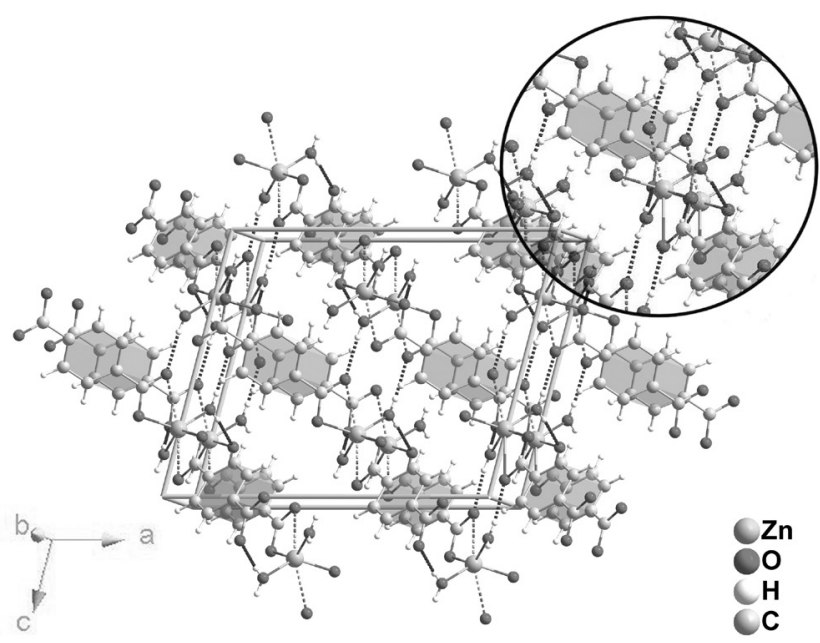

Figura 3. Estrutura supramolecular do $\left[\mathrm{Zn}(\mathrm{BDC})\left(\mathrm{H}_{2} \mathrm{O}\right)_{2}\right]_{n}$, destacando os empacotamentos $\pi-\pi$ (poliedro cinza) e ligações de hidrogênio (região ampliada em pontilhado)
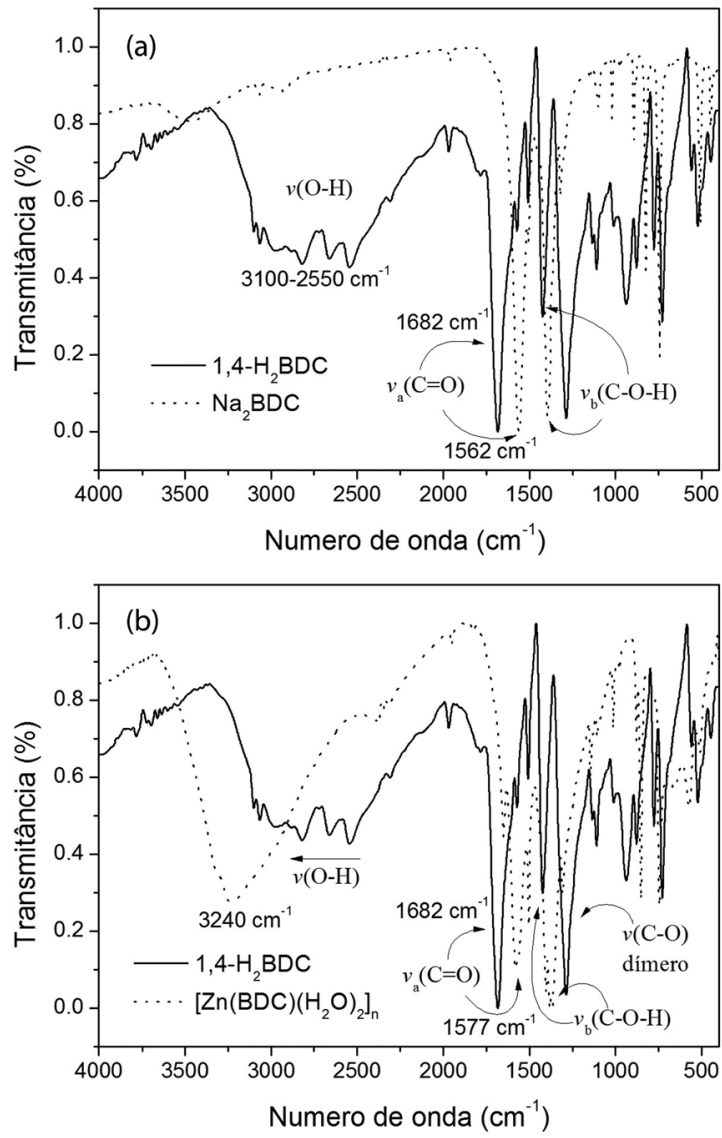

Figura 4. (a) Espectros de infravermelho do 1,4- $\mathrm{H}_{2} \mathrm{BDC}$ (preto) e $\mathrm{Na} \mathrm{B}_{2} \mathrm{BDC}$ (pontilhado); (b) espectros de infravermelho do 1,4- $\mathrm{H}_{2} B D C$ (preto) e do $\left[\mathrm{Zn}(\mathrm{BDC})\left(\mathrm{H}_{2} \mathrm{O}\right)_{2}\right]_{n}$ (pontilhado)

O modo vibracional do estiramento $v(\mathrm{O}-\mathrm{H})$ claramente desaparece quando o $\mathrm{Na}_{2} \mathrm{BDC}$ é formado (Figura 4a). O 1,4- $\mathrm{H}_{2} \mathrm{BDC}$ tem absorções v(O-H) bem definidas centradas em 3103, 3066, 2978, 2816, 2661 e $2546 \mathrm{~cm}^{-1}$, devido às interações intermoleculares das ligações de hidrogênio. ${ }^{41}$ Tem também uma forte banda de absorção em $1682 \mathrm{~cm}^{-1}$ decorrente de $v(\mathrm{C}=\mathrm{O})$ no qual, sob coordenação, desloca para $1562 \mathrm{~cm}^{-1}$ para o $\mathrm{Na}_{2} \mathrm{BDC}$. Um comportamento esperado, visto que a ligação entre o ligante orgânico e o metal faz com que haja uma diminuição na força da ligação da carbonila, deslocando o estiramento para uma menor energia.

Quando o $\left[\mathrm{Zn}(\mathrm{BDC})\left(\mathrm{H}_{2} \mathrm{O}\right)_{2}\right]_{n}$ é formado, o modo vibracional do estiramento $\mathrm{v}(\mathrm{O}-\mathrm{H})$ volta a surgir devido à presença das águas coordenadas na estrutura; entretanto, sob coordenação o estiramento v(O-H) é deslocado para altos números de onda com máximo centrado em $3240 \mathrm{~cm}^{-1}$ comparado às absorções $v(\mathrm{O}-\mathrm{H})$ do 1,4- $\mathrm{H}_{2} \mathrm{BDC}$ (Figura $4 b)$. A forte banda de absorção do $v(\mathrm{C}=\mathrm{O})$ do ligante livre, assim como para o $\mathrm{Na}_{2} \mathrm{BDC}$, desloca para uma menor energia em $1577 \mathrm{~cm}^{-1}$ para o $\left[\mathrm{Zn}(\mathrm{BDC})\left(\mathrm{H}_{2} \mathrm{O}\right)_{2}\right]_{n} \cdot{ }^{32}$ Resultados similares são reportados para outros complexos contendo $1,4-\mathrm{H}_{2} \mathrm{BDC}$ e zinco. ${ }^{42} \mathrm{Os}$ espectros de IV tanto do $\mathrm{Na}_{2} \mathrm{BDC}$ quanto do $\left[\mathrm{Zn}(\mathrm{BDC})\left(\mathrm{H}_{2} \mathrm{O}\right)_{2}\right]_{n}$ têm marcadamente distintas regiões espectrais para os modos vibracionais simétrico de $v_{\mathrm{a}}\left(\mathrm{CO}_{2}^{-}\right)$e assimétrico de $v_{\mathrm{b}}\left(\mathrm{CO}_{2}{ }^{-}\right){ }^{32} \mathrm{~A}$ diferença entre as frequências dos estiramentos $v_{a}\left(\mathrm{CO}_{2}^{-}\right) \mathrm{e} \mathrm{v}_{\mathrm{b}}\left(\mathrm{CO}_{2}^{-}\right)$, correspondendo ao valor $\Delta$, como proposto por Deacon e Phillips ${ }^{43}$ pode indicar o modo como o grupo carboxílico dos compostos podem estar coordenados. Para o $1,4-\mathrm{H}_{2} \mathrm{BDC}$ o valor é $\Delta=258$, para o $\mathrm{Na}_{2} \mathrm{BDC} \Delta=168$ e para o $\left[\mathrm{Zn}(\mathrm{BDC})\left(\mathrm{H}_{2} \mathrm{O}\right)_{2}\right]_{n} \Delta=195$. Como o valor de $\Delta$ do sal de BDC é menor que o do ligante livre, o composto deve estar coordenado pelo tipo quelato, assim como seria esperado para o polímero de coordenação. ${ }^{44}$ $\mathrm{Na}$ descrição da estrutura cristalina foi visto que o $\left[\mathrm{Zn}(\mathrm{BDC})\left(\mathrm{H}_{2} \mathrm{O}\right)_{2}\right]$ ${ }_{n}$ está coordenado pelo modo monodentado, podendo descrever um modo de coordenação semiquelato, devido à presença da ligação $\mathrm{Zn}-\mathrm{O}(2)$ livre para fortes interações de hidrogênio (Figura 2), este fato poderia justificar a diferença entre os valores de $\Delta$ do $\mathrm{Na}_{2} \mathrm{BDC}$ e do $\left[\mathrm{Zn}(\mathrm{BDC})\left(\mathrm{H}_{2} \mathrm{O}\right)_{2}\right]_{n}$.

A Figura 5 ilustra a curva termogravimétrica do $\left[\mathrm{Zn}(\mathrm{BDC})\left(\mathrm{H}_{2} \mathrm{O}\right)_{2}\right]_{n}$. Há marcadamente três perdas de massa distintas. As perdas de massa podem ser interpretadas como: liberação de moléculas de água coordenadas em dois estágios consecutivos, no qual a primeira etapa liberando, em média, uma molécula de água (perda de 6,8\%) entre 140 e $178^{\circ} \mathrm{C}$ e a segunda etapa liberando a outra molécula de água remanescente ligada ao metal zinco (perda também de 6,8\%) entre 179 e $206^{\circ} \mathrm{C}$; liberação do ligante orgânico em um único estágio, correspondendo à quebra, descoordenação do $1,4-\mathrm{H}_{2} \mathrm{BDC}$ e desintegração da estrutura entre 207 e $600{ }^{\circ} \mathrm{C}$ (perda de 55,8\%), levando somente ao óxido metálico. Em alguns casos esta liberação pode ocorrer em duas etapas consecutivas, sendo a primeira correspondente à quebra e descoordenação do ligante orgânico e a segunda, à desintegração da estrutura. ${ }^{45}$

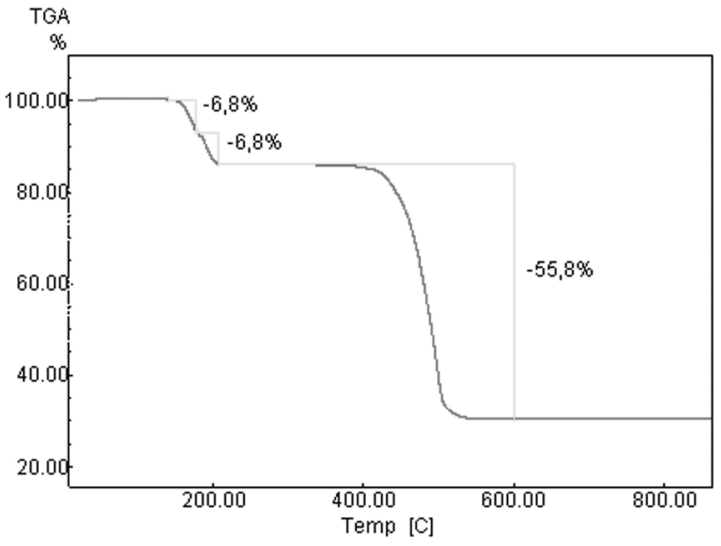

Figura 5. Curva termogravimétrica do $\left[\mathrm{Zn}(\mathrm{BDC})\left(\mathrm{H}_{2} \mathrm{O}\right)_{2}\right]_{n}$

Moléculas de água coordenadas normalmente são liberadas entre 100 e $200^{\circ} \mathrm{C}$, assim como a desintegração do ligante ocorre entre 400 e $600^{\circ} \mathrm{C}$. Dessa forma, os dados termogravimétricos estão de acordo com o que seria esperado para o $\left[\mathrm{Zn}(\mathrm{BDC})\left(\mathrm{H}_{2} \mathrm{O}\right)_{2}\right]_{n}$, podendo-se pro- 
por o mecanismo (Equação 3) para as perdas de massa da molécula.

$\left[\mathrm{ZnC}_{8} \mathrm{H}_{4} \mathrm{O}_{4}\left(\mathrm{H}_{2} \mathrm{O}\right)_{2}\right]_{n} \underset{140-206^{\circ} \mathrm{C}}{\stackrel{-2 \mathrm{nH}_{2} \mathrm{O}}{\longrightarrow}}\left[\mathrm{ZnC}_{8} \mathrm{H}_{4} \mathrm{O}_{4}\right]_{\mathrm{n}} \underset{270-600^{\circ} \mathrm{C}}{\stackrel{-n \mathrm{C}_{8} \mathrm{H}_{4} \mathrm{O}_{3}}{2}}[\mathrm{ZnO}]_{n}$

\section{CONCLUSÃO}

A rede metalorgânica $\left[\mathrm{Zn}(\mathrm{BDC})\left(\mathrm{H}_{2} \mathrm{O}\right)_{2}\right]_{n}$, conhecida na literatura, foi obtida por um procedimento sintético rápido, eficiente e reprodutível. Fatores de confiabilidade obtidos através do refinamento de Rietveld confirmaram a obtenção do material pela síntese assistida por micro-ondas durante 10 min de irradiação, fornecendo excelentes cristais com $90 \%$ de rendimento. Análises elementares, espectroscopia por infravermelho e análise termogravimétrica puderam ser eficientemente correlacionadas aos dados cristalográficos da estrutura linear em zig-zag da rede metalorgânica. As interações dos empacotamentos $\pi-\pi$ da cadeia em $1 \mathrm{D}$ sugerem aplicações interessantes à estrutura.

\section{MATERIAL SUPLEMENTAR}

A estrutura supramolecular do $\left[\mathrm{Zn}(\mathrm{BDC})\left(\mathrm{H}_{2} \mathrm{O}\right)_{2}\right]_{n}$ em cores encontra-se disponível gratuitamente em http://quimicanova.sbq.org. br/, na forma de arquivo pdf.

\section{AGRADECIMENTOS}

À técnica E. de F. V. Barros da Central Analítica e ao Prof. E. H. L. Falcão do Departamento de Química Fundamental (UFPE), ao pesquisador F. A. A. Paz e sua aluna P. Silva da Universidade de Aveiro, Portugal. Ao CNPq, CAPES, RENAMI e INCT-INAMI pelo suporte financeiro.

\section{REFERÊNCIAS}

1. Rowsell, J. L. C.; Yaghi, O. M.; Microporous Mesoporous Mater. 2004, 73,3 .

2. Janiak, C;. Dalton Trans. 2003, 2781.

3. Papaefstathiou, G. S.; MacGillivray, L. R.; Coord. Chem. Rev. 2003, 246, 169.

4. Yaghi, O. M.; O'Keeffe, M.; Ockwig, N. W.; Chae, H. K.; Eddaoudi, M.; Kim, J.; Nature 2003, 423, 705.

5. Rodrigues, M. O.; Brito-Silva, A. M.; Alves, S.; De Simone, C. A.; Araujo, A. A. S.; de Carvalho, P. H. V.; Santos, S. C. G.; Aragão, K. A. S.; Freire, R. O.; Mesquita, M. E.; Quim. Nova 2009, 32, 286.

6. Rodrigues, M. O.; Paz, F. A.; Freire, R. O.; de Sa, G. F.; Galembeck, A.; Montenegro, M. C.; Araujo, A. N.; Alves, S.; J. Phys. Chem. B 2009, $113,12181$.

7. Lin, Z.; Wragg, D. S.; Morris, R. E.; Chem. Commun. 2006, 2021

8. Silva, P. C.; Valente, A. A.; Rocha, J. O.; Almeida Paz, F. A.; Cryst. Growth Des. 2010, 10, 2025.

9. Lidström, P.; Tierney, J.; Wathey, B.; Westman, J.; Tetrahedron 2001, 57, 9225 .

10. Inada, M.; Tsujimoto, H.; Eguchi, Y.; Enomoto, N.; Hojo, J.; Fuel 2005, $84,1482$.

11. Xu, X.; Bao, Y.; Song, C.; Yang, W.; Liu, J.; Lin, L.; Microporous Mesoporous Mater. 2004, 75, 173.

12. Ni, Z.; Masel, R. I.; J. Am. Chem. Soc. 2006, 128, 12394.

13. Choi, J. Y.; Kim, J.; Jhung, S. H.; Kim, H.-K.; Chang, J.-S.; Chae, H. K.; Bull. Korean Chem. Soc. 2006, 27, 1523.
14. Park, S.-E.; Chang, J.-S.; Hwang, Y. K.; Kim, D. S.; Jhung, S. H.; Hwang, J. S.; Catal. Surv. Asia 2004, 8, 91.

15. Collins, D. J.; Zhou, H. C.; J. Mater. Chem. 2007, 17, 3154.

16. Bastin, L.; Barcia, P. S.; Hurtado, E. J.; Silva, J. A. C.; Rodrigues, A. E.; Chen, B.; J. Phys. Chem. C 2008, 112, 1575.

17. Mueller, U.; Schubert, M.; Teich, F.; Puetter, H.; Schierle-Arndt, K.; Pastre, J.; J. Mater. Chem. 2006, 16, 626.

18. Horike, S.; Dinca, M.; Tamaki, K.; Long, J. R.; J. Am. Chem. Soc. 2008, 130, 5854.

19. Horcajada, P.; Serre, C.; Vallet-Regi, M.; Sebban, M.; Taulelle, F.; Ferey, G.; Angew. Chem., Int. Ed. 2006, 45, 5974.

20. Horcajada, P.; Serre, C.; Maurin, G.; Ramsahye, N. A.; Balas, F.; ValletRegi, M.; Sebban, M.; Taulelle, F.; Ferey, G.; J. Am. Chem. Soc. 2008, 130, 6774.

21. Chen, B. L.; Liang, C. D.; Yang, J.; Contreras, D. S.; Clancy, Y. L.; Lobkovsky, E. B.; Yaghi, O. M.; Dai, S.; Angew. Chem., Int. Ed. 2006, 45, 1390

22. Zhou, Y. Y.; Yan, X. P.; Kim, K. N.; Wang, S. W.; Liu, M. G.; J. Chromatogr., A 2006, 1116, 172.

23. Wang, F. Q.; Zheng, X. J.; Wan, Y. H.; Sun, C. Y.; Wang, Z. M.; Wang, K. Z.; Jin, L. P.; Inorg. Chem. 2007, 46, 2956.

24. Tranchemontagne, D. J.; Hunt, J. R.; Yaghi, O. M.; Tetrahedron 2008 , $64,8553$.

25. Britt, D.; Tranchemontagne, D.; Yaghi, O. M.; Proc. Natl. Acad. Sci. U.S.A. 2008, 105, 11623.

26. Eddaoudi, M.; Kim, J.; Rosi, N.; Vodak, D.; Wachter, J.; O’Keeffe, M.; Yaghi, O. M.; Science 2002, 295, 469

27. Eddaoudi, M.; Li, H. L.; Yaghi, O. M.; J. Am. Chem. Soc. 2000, 122, 1391.

28. Guilera, G.; Steed, J. W.; Chem. Commun. 1999, 1563.

29. Li, Z. Q.; Qiu, L. G.; Wang, W.; Xu, T.; Wu, Y.; Jiang, X.; Inorg. Chem. Commun. 2008, 11, 1375.

30. Li, H.; Eddaoudi, M.; O’Keeffe, M.; Yaghi, O. M.; Nature 1999, 402, 276.

31. Edgar, M.; Mitchell, R.; Slawin, A. M. Z.; Lightfoot, P.; Wright, P. A.; Chem.-Eur. J. 2001, 7, 5168.

32. Zhu, L. N.; Zhang, L. Z.; Wang, W. Z.; Liao, D. Z.; Cheng, P.; Jiang, Z. H.; Yan, S. P.; Inorg. Chem. Commun. 2002, 5, 1017.

33. Rietveld, H. M.; J. Appl. Crystallogr. 1969, 2, 65.

34. Larson, A. C.; von Dreele, R. B.; General Structure Analysis System (GSAS), Los Alamos National Laboratory, 2004.

35. Jarvinen, M.; J. Appl. Crystallogr. 1993, 26, 525.

36. Thompson, P.; Cox, D. E.; Hastings, J. B.; J. Appl. Crystallogr. 1987, 20,79 .

37. Young, R. A.; Desai, P.; Arch. Nauki Mater. 1989, 10, 71.

38. Young, R. A.; Wiles, D. B.; J. Appl. Crystallogr. 1982, 15, 430.

39. Zheng, S. L.; Yang, J. H.; Yu, X. L.; Chen, X. M.; Wong, W. T.; Inorg. Chem. 2004, 43, 830

40. Haitao, X.; Jiahe, L.; Jing, Z.; Huizhong, K.; Ruji, W.; Li, Y.; J. Mol. Struct. 2004, 689, 177.

41. Tellez, C. A.; Hollauer, E.; Mondragon, M. A.; Castano, V. M.; Spectrochim. Acta, Part A 2001, 57, 993.

42. Ghermani, N. E.; Morgant, G.; d'Angelo, J.; Desmaele, D.; Fraisse, B.; Bonhomme, F.; Dichi, E.; Sgahier, M.; Polyhedron 2007, 26, 2880.

43. Deacon, G. B.; Phillips, R. J.; Coord. Chem. Rev. 1980, 33, 227.

44. Nakamoto, K.; Infrared and Raman Spectra of Inorganic and Coordination Compounds, Wiley: New Jersey, 2009.

45. Alaerts, L.; Maes, M.; Jacobs, P. A.; Denayer, J. F. M.; De Vos, D. E.; Phys. Chem. Chem. Phys 2008, 10, 2979. 
SÍNTESE HIDROTERMAL ASSISTIDA POR MICRO-ONDAS COMO METODOLOGIA SINTÉTICA EFICIENTE PARA OBTENÇÃO DA REDE METALORGÂNICA $\left[\mathrm{Zn}(\mathrm{BDC})\left(\mathrm{H}_{2} \mathrm{O}\right)_{2}\right]_{n}$

Kaline A. Wanderley* e Severino Alves Júnior

Departamento de Química Fundamental, Universidade Federal de Pernambuco, Av. Luis Freire, s/n, 50590-470 Recife - PE, Brasil Carlos de Oliveira Paiva-Santos

Departamento de Físico-Química, Instituto de Quí́mica, Universidade Estadual Paulista, CP 355, 14801-970 Araraquara - SP, Brasil

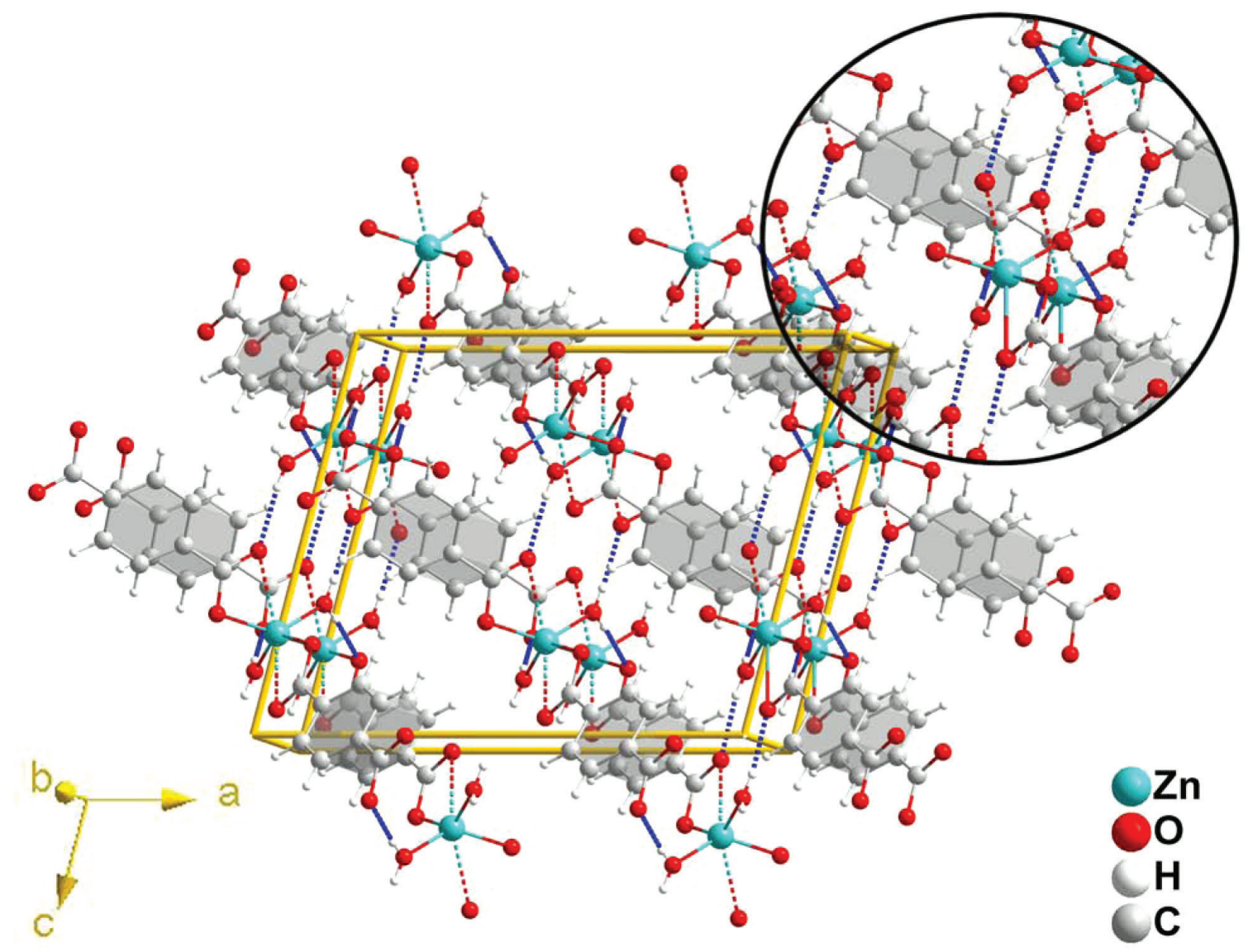

Figura 1S. Estrutura supramolecular do $\left[\mathrm{Zn}(\mathrm{BDC})\left(\mathrm{H}_{2} \mathrm{O}\right)_{2}\right]_{n}$, destacando os empacotamentos $\pi$ - $\pi$ (poliedro cinza) e ligações de hidrogênio (região ampliada em pontilhado) 